\title{
BIOFILM FORMATION IN MALASSEZIA PACHYDERMATIS STRAINS ISOLATED FROM DOGS DECREASES SUSCEPTIBILITY TO KETOCONAZOLE AND ITRACONAZOLE
}

\author{
Ákos JerZSELE ${ }^{1 *}$, Béla GYETVAI ${ }^{2}$, István CSERE $^{3}$ and Péter GÁLFI ${ }^{1}$ \\ ${ }^{1}$ Department of Pharmacology and Toxicology, Faculty of Veterinary Sciences, \\ Szent István University, István u. 2, H-1078 Budapest, Hungary; ${ }^{2}$ Alpha-Vet Veterinary Ltd., \\ Székesfehérvár, Hungary; ${ }^{3}$ Tiflovet Ltd., Budapest, Hungary
}

(Received 14 March 2014; accepted 25 June 2014)

\begin{abstract}
Malassezia pachydermatis is a commonly isolated yeast in veterinary dermatology that can produce biofilms in vitro and in vivo, lowering its susceptibility to antimicrobial drugs. The aim of this study was to determine and compare the in vitro susceptibility of planktonic cells and biofilms of $M$. pachydermatis isolates to ketoconazole and itraconazole. The presence of biofilm formation was confirmed by crystal violet staining and absorbance measurement at $595 \mathrm{~nm}$ wavelength, and by a scanning electron microscopy method. Cell viability was determined by the Celltiter 96 Aqueous One solution assay containing a water-soluble tetrazolium compound (MTS) with absorbance measurement at $490 \mathrm{~nm}$. Planktonic cell minimum inhibitory concentrations (MICs) and minimum fungicidal concentrations (MFCs) of ketoconazole and itraconazole were very low: $\mathrm{MIC}_{90}$ and $\mathrm{MFC}_{90}$ were 0.032 and $0.125 \mu \mathrm{g} / \mathrm{ml}$ for ketoconazole, while 0.063 and $0.25 \mu \mathrm{g} / \mathrm{ml}$ for itraconazole, respectively. Also, the half maximal effective concentrations $\left(\mathrm{EC}_{50}\right)$ of itraconazole were higher for planktonic cells and biofilms compared to ketoconazole. The $\mathrm{EC}_{50}$ values of ketoconazole were 18-169 times higher and those of itraconazole 13-124 times higher for biofilms than for planktonic cells. Biofilm $\mathrm{EC}_{50}$ levels exceeded MICs 103-2060 times for ketoconazole and 84-1400 times for itraconazole. No significant difference was found between these values of the two substances. In conclusion, biofilms of all examined M. pachydermatis strains were much less susceptible to ketoconazole and itraconazole than their planktonic forms.
\end{abstract}

Key words: Biofilm, itraconazole, ketoconazole, Malassezia pachydermatis, planktonic

Malassezia pachydermatis is a frequently isolated commensalistic yeast in veterinary dermatology (Bond, 2010; Outerbridge, 2006). It is a member of the normal skin flora, but can cause secondary dermatitis or external otitis in dogs and

*Corresponding author; E-mail: Jerzsele.Akos@aotk.szie.hu; Phone: 0036 (1) 478-4170; Fax: 0036 (1) 478-4172 
cats with atopic dermatitis, cutaneous food adverse reactions, ectoparasite infestations, immunosuppression, primary keratinisation defects or endocrinopathies (Bond, 2010; Outerbridge, 2006). Common treatments for Malassezia dermatitis and otitis include antifungal agents like polyenes, azoles, allylamines or chlorhexidine. Antifungals belonging to the azole group are one of the most effective and the most frequently used drugs in the management of the infection, ketoconazole and itraconazole being the most active against this microorganism with very low MICs (Brito et al., 2007; Bond, 2010; Jesus et al., 2011; Jerzsele et al., 2013). However, yeasts including malasseziae can produce biofilms in vitro (Cannizzo et al., 2007; Figueredo et al., 2012), decreasing susceptibility to the antimicrobial drugs used. It was reported that Candida albicans, Cryptococcus neoformans, Aspergillus fumigatus, Pneumocystis spp. and Coccidioides immitis might also be able to produce fungal biofilms in humans (Cannizzo et al., 2007; Martinez and Fries, 2010). Biofilms are extracellular matrices produced by microorganisms (primarily bacteria and fungi) which help the pathogens attach to viable and non-viable surfaces (Van Minnebruggen et al., 2010). Biofilm formation in Candida albicans resulted in a 30- to 2000-fold decrease in sensitivity to certain antifungals including ketoconazole (Martinez and Fries, 2010; Vandeputte et al., 2012). Figueredo et al. (2013) reported that M. pachydermatis biofilms exhibited high-level resistance to several antifungals including azoles, at least 3 dilutions higher as compared to planktonic cells.

The aim of this study was to determine and compare the in vitro susceptibility of planktonic cells and biofilms of $M$. pachydermatis strains of canine origin to ketoconazole and itraconazole.

\section{Materials and methods}

Malassezia pachydermatis isolates

Sixteen strains of $M$. pachydermatis were examined. All of the strains were isolated between 2009 and 2011 in Hungary from dogs suffering from clinical signs of external otitis or superficial dermatitis. Clinical specimens were obtained using a sterile dry cotton swab. Malasseziae were identified on the basis of colony morphology on Sabouraud agar, microscopic identification and growth on agar without lipid supplementation (Guillot et al., 1996). The strains were stored at $-80{ }^{\circ} \mathrm{C}$ in Sabouraud dextrose broth (Biolab Co. Ltd., Budapest, Hungary) and $20 \%$ glycerol.

Biofilm production and planktonic cells of $\mathrm{M}$. pachydermatis

Strains grown in Sabouraud dextrose liquid broth at $32{ }^{\circ} \mathrm{C}$ for $72 \mathrm{~h}$ were centrifuged at $3000 \mathrm{~g}$ for $10 \mathrm{~min}$ and resuspended in physiological saline. The 
optical density of the suspension at $600 \mathrm{~nm}$ was set to 0.1 resulting in an approx. $10^{6}$ colony forming units $(\mathrm{CFU}) / \mathrm{ml}$ viable cell count confirmed by agar plate counting.

Biofilm production and subsequent evaluation was carried out according to the method of Hawser and Douglas (1994), with some modifications as recommended by Nett et al. (2011). Briefly, a sterile, disposable silicone elastomer Foley catheter was cut into standardised segments of $10 \mathrm{~mm}$. The surface of these catheters was scraped with sterile, gamma-sterilised scalpel blades to enhance the attachment of yeast cells. Twenty-five $\mu 1$ of the yeast suspension $\left(10^{6} \mathrm{CFU} / \mathrm{ml}\right)$ was pipetted on catheter segment surfaces, put in a thermostat and incubated at $32{ }^{\circ} \mathrm{C}$ for $1 \mathrm{~h}$ (attachment phase) to facilitate adherence of the yeast cells. The segments were submerged in $1 \mathrm{ml}$ of Sabouraud broth in a 24-well microplate (Corning, California, USA) and incubated at $32{ }^{\circ} \mathrm{C}$ for $8 \mathrm{~h}$. After $8 \mathrm{~h}$ the medium was replaced by Sabouraud broth containing different concentrations of ketoconazole (Sigma, St. Louis, USA) or itraconazole (Sigma, St. Louis, USA). Stock solutions of the azoles were prepared in dimethyl sulphoxide (DMSO) and the final concentrations in the microplates were set in the range of 0.004 to $32 \mu \mathrm{g} / \mathrm{ml}$ for both drugs. Fungal suspensions containing the antifungals were placed in triplicates in a thermostat and incubated at $32{ }^{\circ} \mathrm{C}$ for $72 \mathrm{~h}$. By omitting the placement of cells on catheter segments and the attachment phase, they grew in the planktonic form.

The presence of biofilm formation was confirmed by crystal violet staining and absorbance measurement at $595 \mathrm{~nm}$ wavelength (Jin et al., 2003) and a scanning electron microscopy (SEM) method described by Figueredo et al. (2012) utilising a ZEISS EVO MA10 scanning electron microscope. The samples were dehydrated with increasing concentrations of ethanol $(20 \%, 40 \%, 50 \%, 70 \%$ and $90 \%$ ), then coated with gold with a high-vacuum evaporator.

Determination of half maximal effective concentrations $\left(E C_{50}\right)$ and MFC values of ketoconazole and itraconazole

Cell viability was determined by the Celltiter 96 Aqueous One solution assay (Promega, Madison, USA), containing a water-soluble tetrazolium compound (MTS). Formazan produced by viable yeasts was measured by absorbance at $490 \mathrm{~nm}$ and was directly proportional to the number of living cells. Doseresponse curves were used to determine $\mathrm{EC}_{50}$ values (effective concentration $50 \%$ ) of ketoconazole and itraconazole (Hawser and Douglas, 1994; Nett et al., 2011). All assays were performed in triplicates. For planktonic cells, also the minimum inhibitory concentration (MIC) and the minimum fungicidal concentration (MFC) of the drugs were determined. The threshold of resistance was $\leq 0.125 \mu \mathrm{g} / \mathrm{ml}$ in the case of both drugs according to the CLSI (Clinical Laboratory Standards Institute) guidelines. MFC was determined by agar plate CFU counting and defined as the concentration of the drug that decreased viable cell 
number by $99.9 \%$. $\mathrm{MIC}_{90}$ and $\mathrm{MFC}_{90}$ were calculated as the concentrations of drugs that inhibit/kill $99.9 \%$ of fungi in $90 \%$ of the strains investigated.

\section{Statistical analysis}

The paired Student's $t$-test was used to evaluate the differences between MIC, MFC and $\mathrm{EC}_{50}$ mean values of the two antifungal agents. A value of $\mathrm{P}<$ 0.05 was considered to be statistically significant.

\section{Results}

All of the tested 16 M. pachydermatis isolates were able to produce biofilms on the catheter surface, as confirmed by crystal violet staining and SEM. A SEM picture of an 8 hours old M. pachydermatis biofilm is shown in Fig. 1.

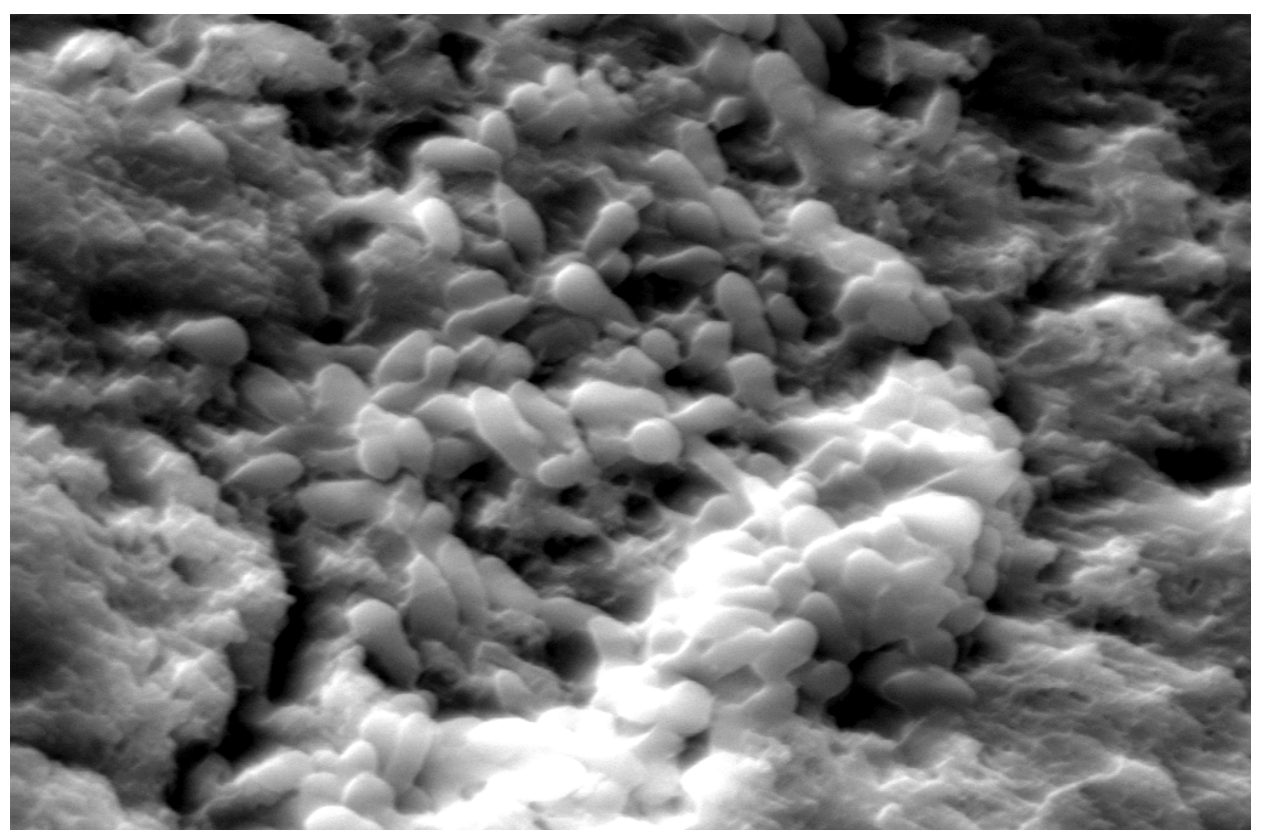

Fig. 1. Scanning electron micrograph of an 8 hours old Malassezia pachydermatis biofilm (Zeiss Evo MA10; magnification: $\times 2500$ )

Planktonic cells were much more sensitive than biofilms to both of the drugs tested. The susceptibility of the investigated M. pachydermatis strains to ketoconazole and itraconazole in biofilms and free-floating planktonic cells is shown in Table 1. MFC/MIC ratios were between 2 and 4, indicating excellent in vitro fungicidal effect of the azoles against $M$. pachydermatis. $\mathrm{MIC}_{90}$ and $\mathrm{MFC}_{90}$ 


\section{Table 1}

Minimum inhibitory concentration (MIC), minimum fungicidal concentration (MFC) and $\mathrm{EC}_{50}$ (concentration able to reduce formazan formation by $50 \%$ ) values of ketoconazole and itraconazole in planktonic cells and biofilms of Malassezia pachydermatis isolated from dogs

\begin{tabular}{|c|c|c|c|c|c|c|c|c|c|c|}
\hline \multirow{3}{*}{ Strain No. } & \multicolumn{5}{|c|}{ Ketoconazole } & \multicolumn{5}{|c|}{ Itraconazole } \\
\hline & \multicolumn{3}{|c|}{ Planktonic cells } & \multirow{2}{*}{$\begin{array}{c}\text { Biofilm } \\
\mathrm{EC}_{50}\end{array}$} & \multirow{2}{*}{$\begin{array}{c}\text { Biofilm EC } \mathrm{EC}_{50} / \\
\text { Planktonic } \\
\mathrm{EC}_{50}\end{array}$} & \multicolumn{3}{|c|}{ Planktonic cells } & \multirow{2}{*}{$\begin{array}{c}\text { Biofilm } \\
\mathrm{EC}_{50}\end{array}$} & \multirow{2}{*}{$\begin{array}{c}\text { Biofilm } \mathrm{EC}_{50} \\
\text { Planktonic } \\
\mathrm{EC}_{50}\end{array}$} \\
\hline & $\mathrm{EC}_{50}$ & MIC & MFC & & & $\mathrm{EC}_{50}$ & MIC & MFC & & \\
\hline 1 & 0.09 & 0.016 & 0.063 & 2.06 & 23 & 0.09 & 0.032 & 0.125 & 2.7 & 30 \\
\hline 2 & 0.06 & 0.008 & 0.032 & 2.68 & 41 & 0.11 & 0.016 & 0.063 & 2.64 & 24 \\
\hline 3 & 0.11 & 0.016 & 0.063 & 1.85 & 18 & 0.17 & 0.016 & 0.063 & 2.23 & 13 \\
\hline 4 & 0.05 & 0.004 & 0.016 & 2.47 & 51 & 0.20 & 0.032 & 0.125 & 3.1 & 16 \\
\hline 5 & 0.19 & 0.008 & 0.032 & 14.42 & 74 & 0.23 & 0.016 & 0.063 & 9.8 & 43 \\
\hline 6 & 0.26 & 0.032 & 0.125 & 13.39 & 52 & 0.40 & 0.032 & 0.125 & 17.9 & 45 \\
\hline 7 & 0.07 & 0.008 & 0.032 & 3.19 & 44 & 0.09 & 0.008 & 0.032 & 4.8 & 53 \\
\hline 8 & 0.10 & 0.008 & 0.032 & 16.48 & 169 & 0.09 & 0.008 & 0.032 & 11.2 & 124 \\
\hline 9 & 0.21 & 0.032 & 0.125 & 12.36 & 59 & 0.17 & 0.016 & 0.063 & 9.95 & 59 \\
\hline 10 & 0.11 & 0.016 & 0.063 & 4.64 & 41 & 0.18 & 0.032 & 0.125 & 11.3 & 63 \\
\hline 11 & 0.34 & 0.032 & 0.125 & 8.76 & 26 & 0.39 & 0.032 & 0.125 & 9.67 & 25 \\
\hline 12 & 0.26 & 0.063 & 0.25 & 6.49 & 25 & 0.33 & 0.063 & 0.25 & 8.9 & 27 \\
\hline 13 & 0.07 & 0.016 & 0.063 & 2.78 & 38 & 0.10 & 0.016 & 0.063 & 4.72 & 47 \\
\hline 14 & 0.09 & 0.008 & 0.032 & 4.02 & 45 & 0.15 & 0.032 & 0.063 & 6.87 & 46 \\
\hline 15 & 0.25 & 0.032 & 0.125 & 10.82 & 43 & 0.35 & 0.063 & 0.25 & 14.3 & 41 \\
\hline 16 & 0.22 & 0.016 & 0.063 & 9.99 & 46 & 0.47 & 0.125 & 0.25 & 13.76 & 29 \\
\hline Mean \pm SD & $0.16 \pm 0.09$ & & & $7.27 \pm 5.01$ & $49.5 \pm 34.9$ & $0.22 \pm 0.13$ & & & $8.37 \pm 4.74$ & $42.7 \pm 26.3$ \\
\hline
\end{tabular}


JERZSELE et al.

for ketoconazole were 0.032 and $0.125 \mu \mathrm{g} / \mathrm{ml}$, while for itraconazole 0.063 and $0.25 \mu \mathrm{g} / \mathrm{ml}$, respectively. Although these values proved to be higher in the case of ketoconazole, differences in individual sensitivity were without statistical significance $(\mathrm{P}>0.05)$. Also, the mean $\mathrm{EC}_{50}$ values for itraconazole were higher for planktonic cells and biofilms compared to ketoconazole but without significant differences $(\mathrm{P}>0.05)$. $\mathrm{EC}_{50}$ values of ketoconazole were 18-169 times higher, while those of itraconazole 13-124 times higher for biofilms than for planktonic cells. Biofilm $\mathrm{EC}_{50}$ levels exceeded the MICs 103-060 times for ketoconazole and 841400 times for itraconazole. No significant difference was found between these values for the two substances.

\section{Discussion}

The MICs and MFCs of ketoconazole and itraconazole were very low as expected from previous studies (Jesus et al., 2011; Cafarchia et al., 2012), and no resistant strain was found to either of the azoles according to the CLSI breakpoints, similarly as reported in earlier studies (Brito et al., 2007). Figueredo et al. (2013) reported low MICs for ketoconazole and itraconazole, with higher MICs for ketoconazole. In our study, ketoconazole was more effective against both planktonic cells or biofilms. In our study, biofilm $\mathrm{EC}_{50}$ levels exceeded the MICs 103-2060 times for ketoconazole and 84-1400 times for itraconazole. In the study of Figueredo et al. (2013), although using a different methodology, $M$. pachydermatis biofilm MICs were approx. 8 times higher than those of planktonic cells. In our study, however, the $\mathrm{EC}_{50}$ values of ketoconazole and itraconazole were 18-169 and 13-124 times higher, respectively. These results show trends similar to those observed in earlier studies performed on Candida albicans strains in human medicine, where a 30- to 2000 -fold higher $\mathrm{EC}_{50}$ was found as compared to the relevant MICs (Hawser and Douglas, 1995). The M. pachydermatis isolates examined in our study were more susceptible to ketoconazole and itraconazole than Candida yeasts with lower MICs and $\mathrm{EC}_{50} \mathrm{~s}$, as has also been demonstrated by Brito et al. (2007).

The primary clinical significance of biofilm formation of yeasts in human medicine is their ability to colonise intravenous catheters and cause bloodstream infections (Leonidou and Gogos, 2010). The fungal species most commonly associated with these infections belong to the Candida genus, although Malassezia spp. are frequently isolated in neonatal intensive care units (Van Belkum et al., 1994). There are no reports on M. pachydermatis causing septicaemia in veterinary medicine. However, its occurrence in veterinary dermatology is common (Outerbridge, 2006). According to Cafarchia et al. (2012), dogs with skin lesions harboured strains of $M$. pachydermatis less susceptible to azoles in vitro than dogs without skin lesions. These observations, along with the results of our study, suggest that secondary Malassezia infections need a special approach for 
evaluating in vitro susceptibility, and emphasise the importance of standardised methods for testing $M$. pachydermatis strains in veterinary medicine (Hector, 2005; Cafarchia et al., 2012).

In conclusion, all M. pachydermatis strains investigated in this study produced biofilms in vitro. Biofilms of all of the strains were much less susceptible to ketoconazole and itraconazole than their planktonic forms as demonstrated by the respective $\mathrm{EC}_{50}$ values.

\section{Acknowledgements}

The study was sponsored by the TÁMOP-4.2.2.B-10/1 and TÁMOP-4.2.1.B-11/2/ KMR-2011-0003 research projects.

\section{References}

Bond, R. (2010): Superficial veterinary mycoses. Clin. Dermatol. 28, 226-236.

Brito, E. H. S., Fontenelle, R. O. S., Brilhante, R. S. N., Cordeiro, R. A., Soares Júnior, F. A., Monteiro, A. J., Sidrim, J. J. C. and Rocha, M. F. G. (2007): Phenotypic characterization and in vitro antifungal sensitivity of Candida spp. and Malassezia pachydermatis strains from dogs. Vet. J. 174, 147-153.

Cafarchia, C., Figueredo, L. A., Iatta, R., Montagna, M. T. and Otranto, D. (2012): In vitro antifungal susceptibility of Malassezia pachydermatis from dogs with and without skin lesions. Vet. Microbiol. 155, 395-398.

Cannizzo, F. T., Eraso, E., Ezkurra, P. A., Villar-Vidal, M., Bollo, E., Castella, G., Cabanes, F. J., Vidotto, V. and Quindos, G. (2007): Biofilm development by clinical isolates of Malassezia pachydermatis. Med. Mycol. 45, 357-361.

Figueredo, L. A., Cafarchia, C., Desantis, S. and Otranto, D. (2012): Biofilm formation of Malassezia pachydermatis from dogs. Vet. Microbiol. 160, 126-131.

Figueredo, L. A., Cafarchia, C. and Otranto, D. (2013): Antifungal susceptibility of Malassezia pachydermatis biofilm. Med. Mycol. 51, 863-867.

Guillot, J., Gueho, E., Lesourd, M., Midgley, G., Chevrier, G. and Dupont, B. (1996): Identification of Malassezia species. A practical approach. J. Mycol. Med. 69, 103-110.

Hawser, S. P. and Douglas, L. J. (1994): Biofilm formation by Candida species on the surface of catheter materials in vitro. Infect. and Immun. 62, 915-921.

Hawser, P. S. and Douglas, L. J. (1995): Resistance of Candida albicans biofilms to antifungal agents in vitro. Antimicrob. Agents Chemother. 39, 2128-2131.

Hector, R. F. (2005): An overview of antifungal drugs and their use for treatment of deep and superficial mycoses in animals. Clin. Tech. Small. An. P. 20, 240-249.

Jerzsele, Á., Balázs, B., Kálmánfi, E., Lajos, Z., Gálfi, P. and Gyetvai, B. (2013): In vitro susceptibility of Malassezia pachydermatis strains isolated from dogs and cats [in Hungarian, with English abstract]. Magyar Állatorvosok Lapja 135, 351-356.

Jesus, F. P., Lautert, C., Zanette, R. A., Mahl, D. L., Azevedo, M. I., Machado, M. L., Dutra, V., Botton, S. A., Alves, S. H. and Santurio, J. M. (2011): In vitro susceptibility of fluconazole-susceptible and -resistant isolates of Malassezia pachydermatis against azoles. Vet. Microbiol. 152, 161-164.

Jin, Y., Yip, H. K., Samaranayake, Y. H., Yau, J. Y. and Samaranayake, L. P. (2003): Biofilmforming ability of Candida albicans is unlikely to contribute to high levels of oral yeast carriage in cases of human immunodeficiency virus infection. J. Clin. Microbiol. 41, 2961-2967. 
Leonidou, L. and Gogos, C. A. (2010): Catheter-related bloodstream infections: catheter management according to pathogen. Int. J. Anitmicrob. Ag. 36S, S26-S32.

Martinez, L. R. and Fries, B. C. (2010): Fungal biofilms: relevance in the setting of human disease. Curr. Fungal Infect. Rep. 4, 266-275.

Nett, J. E., Cain, M. T., Crawford, K. and Andes, D. R. (2011): Optimizing a Candida biofilm microtiter plate model for measurement of antifungal susceptibility by tetrazolium salt assay. J. Clin. Microbiol. 49, 1426-1433.

Outerbridge, C. (2006): Mycologic disorders of the skin. Clin. Tech. Small An. P. 21, 128-134.

Van Belkum, A., Boekhout, T. and Bosboom, R. (1994): Monitoring spread of Malassezia infections in a neonatal intensive care unit by a PCR-mediated genetic typing. J. Clin. Microbiol. 32, 2528-2532.

Vandeputte, P., Ferrari, S. and Coste, A. T. (2012): Antifungal resistance and new strategies to control fungal infections. Int. J. Clin. Microbiol., Article ID 713687, 26 pages. doi:10.1155/ 2012/713687

Van Minnebruggen, G., François, I. E. J. A., Cammue, B. P. A., Thevissen, K., Vroome, V., Borgersand, M. and Shroot, B. (2010): A general overview on past, present and future antimycotics. The Open Mycol. J. 4, 22-32. 